\title{
Assessment of bias-adjusted $\mathbf{P M}_{2.5}$ air quality forecasts over the continental United States during 2007
}

\author{
D. Kang ${ }^{1}$, R. Mathur ${ }^{2}$, and S. Trivikrama Rao ${ }^{2}$ \\ ${ }^{1}$ Computer Science Corporation, Research Triangle Park, 79 T.W. Alexander Drive, NC 27709, USA \\ ${ }^{2}$ Atmospheric Modeling and Analysis Division, National Exposure Research Laboratory, \\ US Environmental Protection Agency, Research Triangle Park, NC, USA
}

Received: 29 October 2009 - Published in Geosci. Model Dev. Discuss.: 8 December 2009

Revised: 7 April 2010 - Accepted: 8 April 2010 - Published: 16 April 2010

\begin{abstract}
To develop fine particulate matter $\left(\mathrm{PM}_{2.5}\right)$ air quality forecasts for the US, a National Air Quality Forecast Capability (NAQFC) system, which linked NOAA's North American Mesoscale (NAM) meteorological model with EPA's Community Multiscale Air Quality (CMAQ) model, was deployed in the developmental mode over the continental United States during 2007. This study investigates the operational use of a bias-adjustment technique called the Kalman Filter Predictor approach for improving the accuracy of the $\mathrm{PM}_{2.5}$ forecasts at monitoring locations. The Kalman Filter Predictor bias-adjustment technique is a recursive algorithm designed to optimally estimate bias-adjustment terms using the information extracted from previous measurements and forecasts.

The bias-adjustment technique is found to improve $\mathrm{PM}_{2.5}$ forecasts (i.e. reduced errors and increased correlation coefficients) for the entire year at almost all locations. The NAQFC tends to overestimate $\mathrm{PM}_{2.5}$ during the cool season and underestimate during the warm season in the eastern part of the continental US domain, but the opposite is true for the Pacific Coast. In the Rocky Mountain region, the NAQFC system overestimates $\mathrm{PM}_{2.5}$ for the whole year. The biasadjusted forecasts can quickly (after 2-3 days' lag) adjust to reflect the transition from one regime to the other. The modest computational requirements and systematic improvements in forecast outputs across all seasons suggest that this technique can be easily adapted to perform bias adjustment for real-time $\mathrm{PM}_{2.5}$ air quality forecasts.
\end{abstract}

Correspondence to: D. Kang

(kang.daiwen@epa.gov)

\section{Introduction}

Ozone $\left(\mathrm{O}_{3}\right)$ and fine particulate matter $\left(\mathrm{PM}_{2.5}\right.$; particles with aerodynamic diameters less than $2.5 \mu \mathrm{m}$ ) in the atmosphere have been a major concern because of their adverse effects on human and ecosystem health. Adverse health effects in humans have been shown to be associated with exposure to elevated ambient $\mathrm{PM}_{2.5}$ levels (e.g., NRC, 1998). $\mathrm{O}_{3}$ and $\mathrm{PM}_{2.5}$ are the two pollutants used in the US to compute the Air Quality Index (AQI), a standardized indicator of air quality conditions at a given location (http://www.airnow.gov); the current AQI standard in the United States is primarily based on daily maximum $8-\mathrm{h} \mathrm{O}_{3}$ and daily mean (24-h average) $\mathrm{PM}_{2.5}$ concentrations. Thus, to develop accurate AQIbased health advisories, it is desirable that air quality forecast systems at least be capable of forecasting these two species well. Real-time $\mathrm{O}_{3}$ forecasts using air quality models have been publicly available in the US for several years over different domains (McHenry et al., 2004; McKeen et al., 2005; Otte et al., 2005; Eder, et al., 2006), while real-time $\mathrm{PM}_{2.5}$ forecasts are mainly in the developmental stage and not available to the general public. The NAQFC (Otte et al., 2005), developed by the National Oceanic and Atmospheric Administration (NOAA) and the US Environmental Protection Agency (EPA) couples NOAA's operational North American Mesoscale (NAM) weather prediction model (Black, 1994; Rogers et al., 1996; http://www.dtcenter.org/wrf-nmm/users) with EPA's Community Multiscale Air Quality (CMAQ) model (Byun and Schere, 2006). It has the capability to provide real-time forecasts for both $\mathrm{O}_{3}$ and $\mathrm{PM}_{2.5}$. The developmental mode model predictions are available for the year of 2007 over the continental US domain, providing a consistent

Published by Copernicus Publications on behalf of the European Geosciences Union. 
and unique data set for performing comprehensive evaluations of bias-corrected pollutant fields.

While it is recognized that $\mathrm{PM}_{2.5}$ pollution results from both primary emissions and secondary formation through complex photochemical and heterogeneous chemical pathways, significant scientific and technical challenges surround the characterization of ambient $\mathrm{PM}_{2.5}$ distributions both through modeling and measurements (e.g., McMurry, 2000; Donahue et al., 2009). The emissions and physical, chemical, and removal processes controlling day-to-day levels of ambient $\mathrm{PM}_{2.5}$ and precursor concentrations also exhibit seasonal variability, resulting in significant spatial and seasonal variability in ambient $\mathrm{PM}_{2.5}$ mass and its chemical composition. Current uncertainties in these individual components pose enormous challenges for developing accurate short-term $\mathrm{PM}_{2.5}$ forecasts (Mathur et al., 2008; Yu et al., 2008). Nevertheless, a need exists for local air quality agencies to provide accurate forecast of $\mathrm{PM}_{2.5}$ concentrations to alert the sensitive population on the onset and duration of unhealthy air associated with elevated $\mathrm{PM}_{2.5}$ levels. To address this need, the utility of $\mathrm{PM}_{2.5}$ forecast guidance obtained from comprehensive atmospheric models can, in the short-term, be improved through post-processing of forecast output with bias-adjustment methods; this is the primary motivation for the analysis presented in this study. It should be noted that post-processing bias-adjustment techniques are routinely used in conjunction with numerical weather prediction models, despite decades of research to improve the formulations in the meteorological models, to develop more accurate forecast products (Glahn and Lowry, 1972; http: //www.weather.gov/mdl/synop/products.php). Given the relatively early state of $\mathrm{PM}_{2.5}$ forecast models and large uncertainties in process representations, the exploration of biasadjustment techniques to improve the usefulness of $\mathrm{PM}_{2.5}$ forecasts is warranted.

Different bias-adjustment (also referred to as biascorrection) techniques have been used for improving surface $\mathrm{O}_{3}$ predictions in recent years (McKeen et al., 2005; Delle Monache et al., 2006; Wilczak et al., 2006; Delle Monache et al., 2008; and Kang et al., 2008). Among these techniques, the Kalman Filter (KF) predictor (hereafter referred to as KF bias-adjustment or simply KF) forecast method yielded the most forecast skill improvement. Kang et al. (2008) presented the application of $\mathrm{KF}$ technique to $\mathrm{O}_{3}$ forecasts over the continental US domain for a three-month period from July to September 2005. While the technique was found to improve the forecast skill for $\mathrm{O}_{3}$, it was not clear if they would be readily applicable for PM forecasts and whether they would yield similar improvements in PM forecast skill. This is primarily due to the fact that unlike $\mathrm{O}_{3}$, elevated $\mathrm{PM}_{2.5}$ concentrations are encountered throughout the year and that significant seasonal biases exist in current models both in the representation of total $\mathrm{PM}_{2.5}$ mass as well as its composition (cf. Mathur et al., 2008; McKeen et al., 2007; Appel et al., 2008). Additionally, the chemical con- stituent contributing to the bias could also vary both spatially and seasonally. Thus, for improved PM forecasts, the biasadjustment techniques should be capable of correcting biases and errors that not only change with time, but that also may have widely varying sources of origin.

In this study, the KF bias-adjustment technique is applied to $\mathrm{PM}_{2.5}$ forecasts for the year of 2007 over the continental US domain. To our knowledge, this is the first comprehensive assessment of the bias-adjustment technique for $\mathrm{PM}_{2.5}$ forecasts. Within the continental US domain, there are about 500 AIRNow sites that report hourly $\mathrm{PM}_{2.5}$ concentrations which are measured using the Tapered Element Oscillating Microbalance (TEOM) method. The year-long forecast period over the continental US has provided a unique data set covering a wide range of atmospheric conditions and a broad $\mathrm{PM}_{2.5}$ concentration range to test the performance of the bias-adjustment technique for $\mathrm{PM}_{2.5}$ forecasts.

The objectives of this study include: (1) apply the KF postprocessing technique to improve skills for real-time $\mathrm{PM}_{2.5}$ forecasts, (2) investigate the spatial and temporal characteristics of this technique when applied to $\mathrm{PM}_{2.5}$ forecasts, and (3) analyze the impact of bias adjustment on forecast errors of different types (e.g., systematic versus unsystematic). Section 2 describes the modeling system, the implementation of the KF bias-adjustment technique, observational data, and evaluation metrics. In Sect. 3 the performance evaluation results and discussions are presented. And the results and conclusions are summarized in Sect. 4.

\section{Experiments and methods}

\subsection{The NAQFC system}

The NAQFC system consists of 3 primary components: (1) the National Weather Service's North American Mesoscale (NAM) model based on the Weather Research Forecast nonhydrostatic mesoscale model (WRFNMM) (http://www.dtcenter.org/wrf-nmm/users) which provides the meteorological and atmospheric dynamic conditions for the Air Quality Forecast (AQF); (2) the US EPA's Community Multiscale Air Quality (CMAQ) (Byun and Schere, 2006) model, which simulates the transport, chemical evolution, and deposition of atmospheric substances; and (3) an interface component (PREMAQ) that processes both the meteorological and emission inputs to conform with the CMAQ grid structure, coordinate system, and input format. The WRF-NMM (version 2.0) covers 1/3 of Northern Hemisphere with central latitude-longitude at N52, W106 (southern central Canada) using $12 \mathrm{~km}$ horizontal grid spacing and rotated latitude-longitude projection with Arakawa E-gridstaggering. There are 60 vertical layers with lowest interface at $38 \mathrm{~m}$ and the model top is set at $2 \mathrm{hPa}$. The CMAQ (version 4.6) domain for $\mathrm{PM}_{2.5}$ forecasts covers the continental US (Fig. 1) using a 12-km horizontal grid spacing 


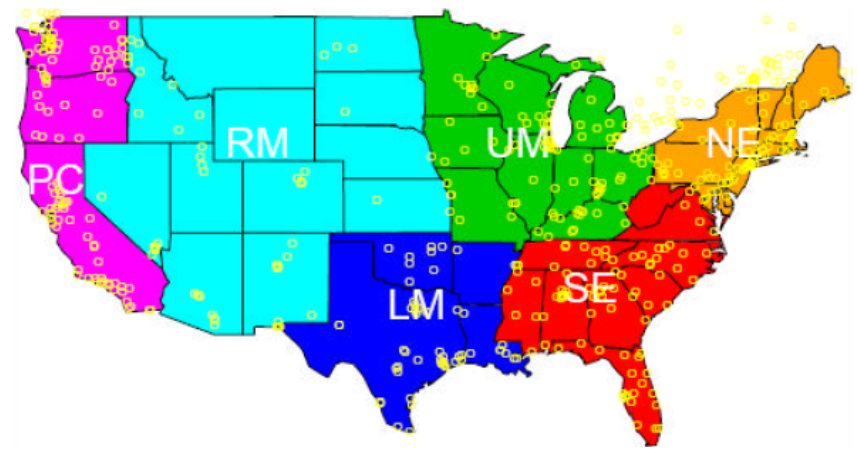

Fig. 1. Analysis sub-regions and monitoring sites (AIRnow network), the horizontal domain is discretized with a $442 \times 26512 \mathrm{~km}$ grid cells. NE: Northeast, SE: southeast, UM: Upper Midwest, LM: Lower Midwest, RM: Rocky Mountains, PC: Pacific Coast.

on the Lambert Conformal map projection and 22 vertical layers of variable thickness set on a sigma coordinate ranging from the surface to $100 \mathrm{hPa}$. Since the $\mathrm{PM}_{2.5}$ forecasts were in the developmental stage, changes or modifications to the AQF components were allowable to accommodate new developments reflecting evolving science. For instance, on 17 September 2007, the treatment for the PBL mixing height scheme in CMAQ was changed from the Turbulence Kinetic Energy (TKE)-based method to the Asymmetric Convective Model-2 (ACM2)-based method, which on average decreased the PBL depth, helping reduce forecast errors for both $\mathrm{O}_{3}$ and $\mathrm{PM}_{2.5}$ in the Pacific Coast region. However, this study does not deal with the impacts of the various changes or modifications to the forecast model; rather, it focused on how the bias-adjustment technique can improve the forecast results over the raw model forecasts. Since the bias-adjustment technique employed in this study is statistical type, it does not involve any modifications in the physical and chemical processes treated in the forecast model.

The emissions inventories used by the AQF system were updated from the US EPA's 2001 national emission inventory to represent the 2007 forecast year (Eder et al., 2009). The biogenic emissions were processed using Biogenic Emission Inventory System (BEIS) version 3.13 (Schwede et al., 2005). Emissions from sea salt, wild fires, and wind-blown dust were not considered for the AQF system, which may contribute to the underestimation of $\mathrm{PM}_{2.5}$ forecasts under some circumstances. The Carbon Bond chemical mechanism (version 4.2) is used to represent the photochemical reactions and AERO3 aerosol module is used to represent aerosol formation and distribution. The chemical fields for CMAQ are initialized using the previous forecast cycle. The primary NAM-CMAQ model forecast for the next 48-h surface-layer $\mathrm{PM}_{2.5}$ is based on the current day's 06:00 UTC cycle, and this is the only cycle available for the developmental $\mathrm{PM}_{2.5}$ forecasts.

\subsection{Observations}

Hourly, near real-time, $\mathrm{PM}_{2.5}$ measurements $\left(\mu \mathrm{g} / \mathrm{m}^{3}\right)$ obtained from EPA's AIRNow program are used in this study (http://www.epa.gov/airnow). All measurements are made using TEOM instruments and concentrations are averaged over hourly intervals from the beginning of one hour to the next. It should be recognized that TEOM measurements are somewhat uncertain and are believed to be lower limits to a "true" value because of volatilization of semivolatile material (ammonium nitrate and organic carbon) in the drying stages of the measurement (Eatough et al., 2003; Grover et al., 2005). Nevertheless, the TEOM measurements are the only real-time hourly $\mathrm{PM}_{2.5}$ observation data available for use in the purpose of this study. About $500 \mathrm{PM}_{2.5}$ monitoring stations are available within the continental US domain (Fig. 1) for the year of 2007. For verification purposes and forecast products, the daily (24-h) mean $\mathrm{PM}_{2.5}$ concentrations are often used.

\subsection{Implementation of the KF bias-adjustment method}

The KF predictor bias-adjustment algorithm (Kalman, 1960) was described in detail by Delle Monache et al. (2006) and a concise description of its implementation was provided by Kang et al. (2008). The specification of the error ratio, a key parameter in the KF approach which determines the relative weighting of observed and forecast values, was previously investigated extensively for $\mathrm{O}_{3}$ forecasts. Even though the optimal error ratios were found to vary across space, the impact of using different optimal values over the model domain on the resultant bias-adjusted $\mathrm{O}_{3}$ predictions was insignificant when compared to using a representative fixed value across all locations (Kang et al., 2008). To test whether the same conclusion is valid for the $\mathrm{PM}_{2.5}$ forecasts, error ratios ranging from 0.01 to 0.10 were selected to perform $\mathrm{PM}_{2.5}$ forecasts for all the sites across the domain over the entire year, and RMSE values were calculated at each site to gauge the impact of spatially different error ratio values on the forecast performance. As shown in Fig. 2, the impact of different error ratio values ranging from 0.04 to 0.10 on the forecast performance is small, and only when the error ratio of 0.01 was used, the RMSE values were relatively larger than using other values. Hence, in this study, we used the same single fixed error ratio value of 0.06 at all the locations for developing bias-adjusted $\mathrm{PM}_{2.5}$ forecasts. In these plots (and also applies to all the boxplots in this paper), the metric is calculated at each site for the specific period and then is presented across all the sites within a region or the entire domain as a box plot, with the lower and upper borders of the box representing the first and third quartiles while the middle line represents the median value.

There are two steps to implement the KF bias-adjustment technique. First, the KF is initialized with the initial estimates of KF parameters as outlined in Kang et al. (2008) and 


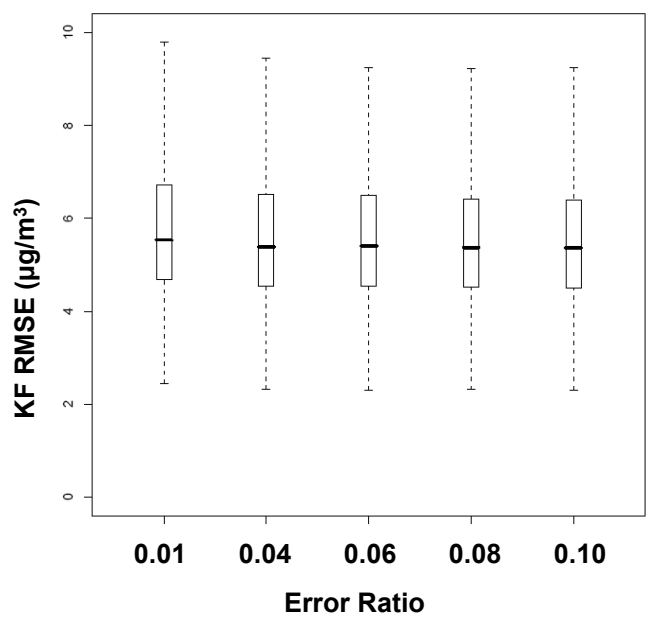

Fig. 2. Impact of error ratios on the performance (RMSE) of Kalman Filter adjusted forecasts for the daily mean $\mathrm{PM}_{2.5}$ concentrations $\left(\mu \mathrm{g} / \mathrm{m}^{3}\right)$.

hourly observations and raw model predictions for the prior 2 days. Then the updated parameters and the third day's raw model forecasts are used to create bias-adjusted forecasts for the 3rd day. All updated KF parameters for each hour and at each site are saved into a file for use in the subsequent $\mathrm{KF}$ run. The KF runs then continue by reading the previous day's KF parameters and observations and raw model predictions from the prior 2 days to generate the next day's bias-adjusted forecasts by combining with the next day's raw model forecasts. Thus, in developing the daily KF forecasts, if data for two consecutive days are missing at a site, the KF will automatically drop this site from future bias-adjustment forecasts; however, if a new site with two consecutive days' data appears in the observation data set, the KF will initialize the site with initial values of KF parameters and generate bias-adjusted forecasts further onward. This implementation is adaptable in real-time to the variable nature of monitoring stations which report hourly observations to the AIRNOW network and can be easily combined with AQF system to produce real-time bias-adjusted forecasts.

\subsection{Verification statistics and spatial-temporal considerations}

To assess the performance of the KF bias-adjusted forecasts, a variety of statistical metrics are used, including Root Mean Square Error (RMSE) and its systematic and unsystematic components, Normalized Mean Error (NME), Mean Bias (MB), Normalized Mean Bias (NMB), correlation coefficient $(r)$, and Index Of Agreement (IOA). For a forecast product, it is also important to evaluate its performance over categorical forecasts (Kang et al., 2005). Two categorical metrics, False Alarm Ratio (FAR) and Hit Rate (H), are used in this study.

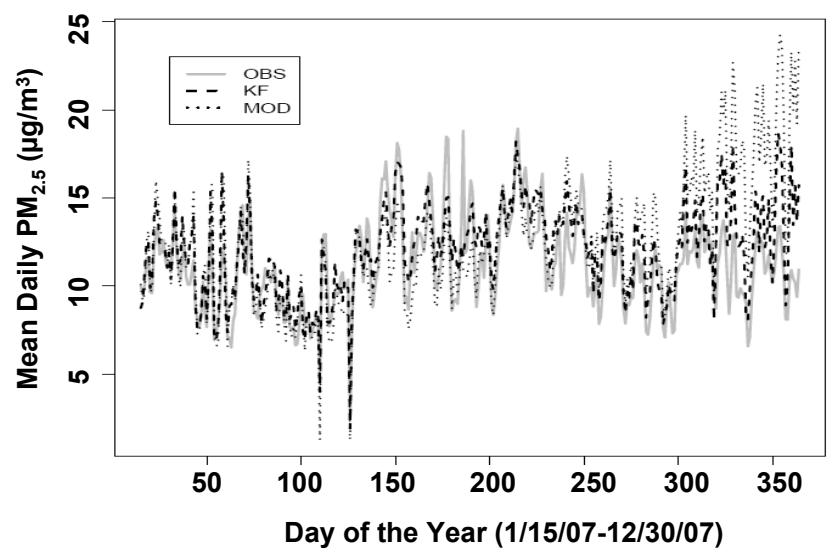

Fig. 3. Time series of observed, raw model forecast, and KF biasadjusted forecast daily mean $\mathrm{PM}_{2.5}\left(\mu \mathrm{g} / \mathrm{m}^{3}\right)$. OBS: observations, KF: Kalman filter bias-adjustment, MOD: raw model.

Since the NAQFC domain covers the continental United States and given large region-to-region differences in the physical and chemical processes, the continental US domain is divided into six subregions following US state boundaries to facilitate the performance evaluations (Fig. 1). The four easternmost subregions, northeast (NE), southeast (SE), upper Midwest (UM), and lower Midwest (LM), are based on $\mathrm{O}_{3}$ and other chemical species climatology that identified areas of homogeneous variability using principal component analysis (Eder et al., 1993; Gego et al., 2005). The domainwide statistics are calculated using all the observations available within the domain.

Figure 3 presents comparisons of time series of the domain-wide daily average observed, raw model forecasts, and KF bias-adjustment forecasts of $\mathrm{PM}_{2.5}$ concentrations during 2007. As shown in Fig. 3, the raw model tends to overpredict during the cool season (before mid-April and after August) and underpredict during the warm season (midApril to end of August) when compared with observations. To facilitate the temporal performance evaluations, the time series are divided into cool season (from January to 20 April and from September to December) and warm season (from 21 April to 31 August).

\section{Results}

\subsection{General performance}

It is evident in Fig. 3 that the raw model has overestimated $\mathrm{PM}_{2.5}$ concentrations on average during the cool season, especially during the period from September to December. During the warm season, the raw model significantly underestimated, and the KF predictions were well above the raw model predictions and much closer to the observations. From late July to early September, the raw model underwent 


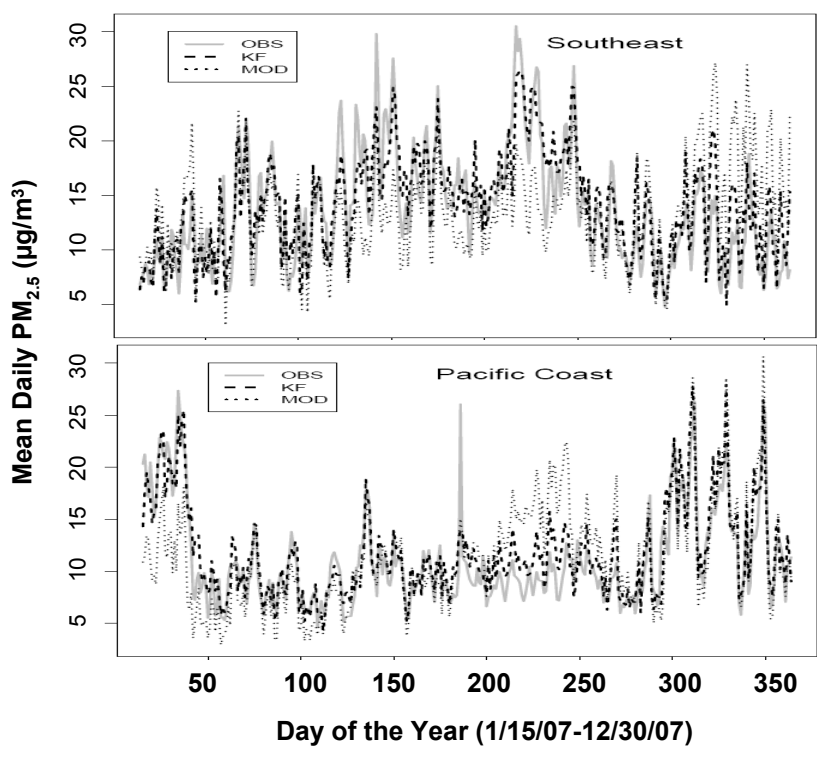

Fig. 4. Time series of observed, raw model forecast, and KF biasadjusted forecast daily mean $\mathrm{PM}_{2.5}\left(\mu \mathrm{g} / \mathrm{m}^{3}\right)$ at Southeast and $\mathrm{Pa}$ cific Coast.

a transitional period from underestimation to overestimation. From early September until the end of the year, the overestimation of the raw model became larger. This is partially attributed to the change of the PBL mixing scheme for CMAQ on 17 September, as mentioned before. Nevertheless, the KF bias-adjustment technique could quickly respond to the transitions from one regime to another and tracked the observations well in the time series. Since Fig. 3 presents the aggregate results for the entire domain, some important information may be hidden due to smoothing during the averaging process. Figure 4 displays same time series as Fig. 3 for two representative sub-regions: Southeast and Pacific Coast. The time series for the Southeast resembles that of the domain, with raw model overestimating during cool season and underestimating during the warm season. However, the underestimation during the warm season is more pronounced for the Southeast than for the entire domain. The time series of the Pacific Coast reveals a completely different story, in which the raw model generally over-predicted during the cool season and under-predicted during the warm season. The over-prediction was much stronger at the beginning of the year (January and early February) than that over the rest of cool season. The over-prediction for the later cool season (from September to December) was reduced, and during most times the raw model could reproduce the observations quite well. The performance change of the raw model during cool season is attributable to the adoption of the new PBL mixing height parameterization when the TKE-based PBL height was replaced by the ACM2-based PBL height. The ACM2-based PBL height generally leads to higher $\mathrm{PM}_{2.5}$ concentrations than the TKE-based PBL height since the

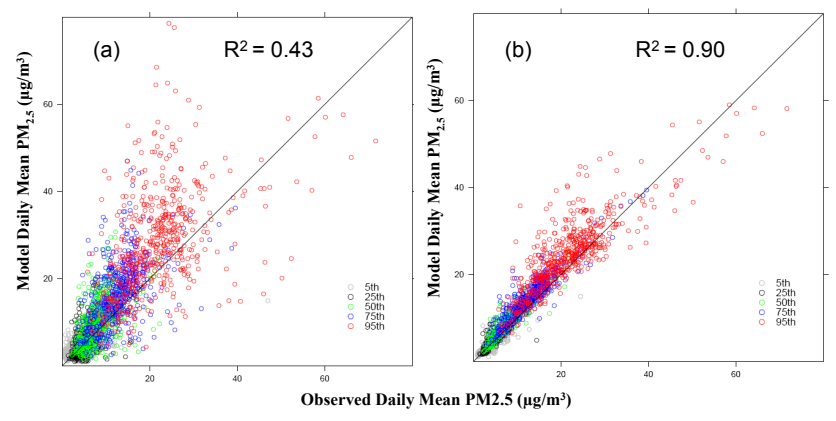

Fig. 5. Scatterplots between forecasts and observations for selected percentiles for the daily mean $\mathrm{PM}_{2.5}$ concentrations $\left(\mu \mathrm{g} / \mathrm{m}^{3}\right)$ : (a) raw model forecasts, (b) Kalman filter-adjusted forecasts.

ACM2-based PBL height is generally lower than the TKEbased PBL height. The under-prediction is thus reduced for the west region of the domain during the cool season, but the over-prediction is further aggravated for the eastern part of the domain during the same period. Nonetheless, the time series of the KF bias-adjusted predictions tracked the observed time series better than the raw model predictions.

To further investigate the performance of the KF biasadjusted forecasts and compare with the raw model forecasts, Fig. 5 displays the scatter plots of forecast and observed values across various percentiles for the daily mean $\mathrm{PM}_{2.5}$ for all the stations within the continental US domain. Following Mathur et al. (2008), at each site the time series of both measured and model (or KF bias-adjusted model) daily mean $\mathrm{PM}_{2.5}$ over the entire year was examined and percentiles of the distribution over the study period were computed for both modeled and observed values. Scatter plots of specific percentiles of the concentration distributions (e.g., median) of the model and observed time series are then examined to assess the ability of the model to capture the spatial variability in frequency distributions of $\mathrm{PM}_{2.5}$ concentrations across the sites (Mathur et al., 2008). As shown in Fig. 5, compared with the raw model forecasts (left), the KF bias-adjusted forecasts displayed a much better match with the observed distributions as reflected by the reduced scatter about the 1:1 line, especially for the higher percentiles. The overall correlation between model forecasts and observations was greatly improved with the value of $R^{2}$ increasing from 0.43 for the raw model forecasts to 0.90 for the KF bias-adjusted forecasts. Similar improvements in $\mathrm{O}_{3}$ forecasts after the application of the KF bias adjustment were previously reported in Kang et al. (2008).

The ability of the KF bias-adjustment technique to improve the predicted $\mathrm{PM}_{2.5}$ concentration distributions is further illustrated in Fig. 6 which displays the histograms of observed daily mean $\mathrm{PM}_{2.5}$ concentrations along with the fitted probability density functions (PDFs) of daily mean $\mathrm{PM}_{2.5}$ concentrations for the observations, raw model forecasts, and KF bias-adjusted forecasts. Figure 6a displays the overall 

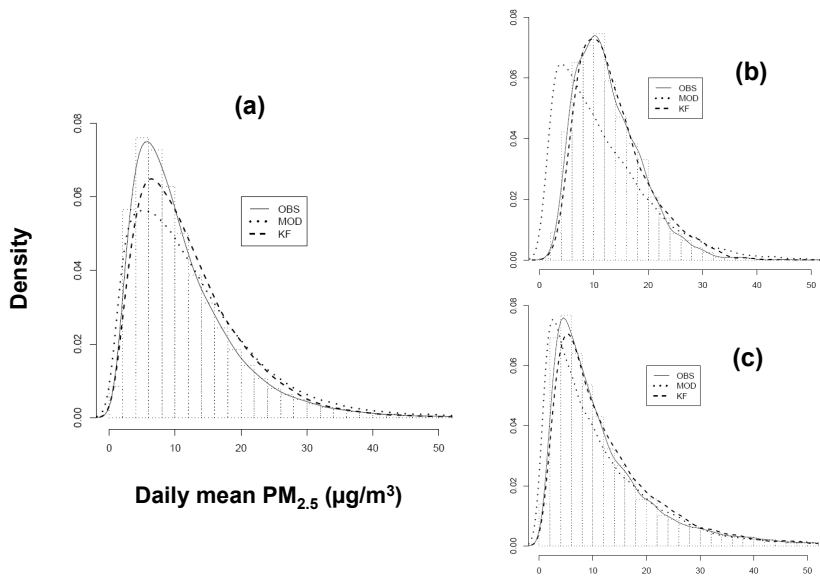

Fig. 6. The histogram of observed and the fitted Gaussian probability density function of observed, raw model forecast, and KF forecast daily mean $\mathrm{PM}_{2.5}$ concentrations $\left(\mu \mathrm{g} / \mathrm{m}^{3}\right)$ : (a) Domain over entire year, (b) LM during warm season, and (c) PC during cool season.

distribution for the entire domain during 2007, while Fig. $6 \mathrm{~b}$ presents the distribution for Lower Midwest during the warm season and Fig. 6c for Pacific Coast during the cool season to typify the sub-regional and seasonal signals. As seen in Fig. 6 , the KF technique brings the PDFs of forecast values much closer to those of the observations. The improvements are more pronounced in the sub-regional and seasonal distribution comparisons illustrated in Fig. $6 \mathrm{~b}$ and c. The distributions of the raw model forecasts for both cases were out of phase compared to those of the observations, especially for Lower Midwest during the warm season. The KF biasadjusted forecasts were able to reproduce the observations very well in both cases.

\subsection{Regional performance}

Tables 1 and 2 present the domain and sub-regional summary of discrete statistics for the raw model and the KF bias-adjusted daily mean $\mathrm{PM}_{2.5}$ forecasts during the cool and warm seasons, respectively. Examination of Table 1 reveals that during the cool season, the RMSE values range from 7.2 to $11.4\left(\mu \mathrm{g} / \mathrm{m}^{3}\right)$ for the raw model forecasts, and from 5.2 to $7.6\left(\mu \mathrm{g} / \mathrm{m}^{3}\right)$ for the KF bias-adjusted forecasts; this translates to about a $20 \%$ reduction in RMSE as a result of the application of the bias adjustment. Similar reductions are also noted for the NME. The MB and NMB indicate that during the cool season, the raw model systematically overpredicted daily mean $\mathrm{PM}_{2.5}$ across all the sub-regions except the Pacific Coast where it under-predicted. The KF biasadjusted forecast reduced NMB values across all the subregions. Correlation coefficients also increased significantly across all the regions as a result of the bias adjustment, with the largest increase in the LM and RM regions. The summary statistics during the warm season (Table 2) indicate compara- ble improvement in the error statistics (RMSE and NME) for the KF bias-adjusted forecasts relative to the raw model. In contrast to the cool season, systematic under-predictions are noted in the warm season raw model $\mathrm{PM}_{2.5}$ forecasts (Mathur et al., 2008). The application of the KF bias adjustment helps reduce both the cool season high bias and the warm season low bias, and also results in consistently improved correlations with measurements across all seasons.

Figure 7 presents comparisons of the distribution of monthly RMSE values of daily mean $\mathrm{PM}_{2.5}$ for the raw model and KF forecasts for the different sub-regions. As seen in Fig. 7, the RMSE values are consistently lower for the KF forecasts relative to those of the raw model across all sub-regions and months. In addition, the error distribution range (the size of the boxes) for the KF forecasts is also much smaller than the raw model forecasts. During OctoberDecember, the raw model forecasts exhibited large RMSE values for both the UM and LM sub-regions (partly attributed to a change in the PBL height parameterization discussed earlier). The KF bias adjustment was able to reduce these large RMSEs significantly. In making comparisons across different regions, it should be noted that the relatively larger spread in RMSE for the RM and PC regions, especially for the raw model forecasts likely resulting from a combination of effects related to complex topography, land-sea breeze transitions in the PC region, greater spatial heterogeneity in emissions, and their impact on chemistry leading to $\mathrm{PM}_{2.5}$ formation and distribution.

Figure 8 presents the spatial distribution of mean biases at each site within the modeling domain for both the cool and warm seasons. As illustrated in Fig. 8a, during the warm season, the raw model predominantly under-predicted at most sites (orange and purple squares) in the eastern part of the domain, over-predicted in the northwest regions and exhibited both over- and under-predictions at sites in California. During the cool season, the raw model generally over-predicted (Fig. 8c) in the east, but under-predictions dominated at sites in western portions of the domain. The application of the KF bias adjustment was able to effectively reduce these biases at more than $90 \%$ of the sites (Fig. 8b and d) to less than $2 \mu \mathrm{g} / \mathrm{m}^{3}$. Even at the sites where absolute values of mean biases were greater than $2 \mu \mathrm{g} / \mathrm{m}^{3}$ for the raw model, the magnitude of the bias was significantly reduced with bias correction.

The forecast skill improvement over space by the KF forecasts over the raw model forecasts is further demonstrated by the IOA as shown in Fig. 9. The IOA increased on average from $8 \%$ (at NE and $\mathrm{UM}$ ) to $30 \%$ at PC during the warm season (Fig. 9a) and from $15 \%$ (at NE and SE) to $28 \%$ at RM during the cool season (Fig. 9b). The domain-wise average IOA values increased by $13 \%$ and $19 \%$ for the warm season and the cool season, respectively. 
Table 1. Regional summary of discrete statistics for raw model and KF bias-adjusted daily mean $\mathrm{PM}_{2.5}$ forecasts during 2007 cool season ( $n$ is the number of records).

\begin{tabular}{lcccccccc}
\hline TYPE & $\mathrm{N}$ & $\begin{array}{c}\text { Obs.mean } \\
\left(\mu \mathrm{g} / \mathrm{m}^{3}\right)\end{array}$ & $\begin{array}{c}\text { Mod.mean } \\
\left(\mu \mathrm{g} / \mathrm{m}^{3}\right)\end{array}$ & $\begin{array}{c}\text { RMSE } \\
\left(\mu \mathrm{g} / \mathrm{m}^{3}\right)\end{array}$ & $\begin{array}{c}\text { NME } \\
(\%)\end{array}$ & $\begin{array}{c}\text { MB } \\
\left(\mu \mathrm{g} / \mathrm{m}^{3}\right)\end{array}$ & $\begin{array}{c}\text { NMB } \\
(\%)\end{array}$ & $r$ \\
\hline Dom-mod & 97243 & 10.54 & 13.08 & 9.6 & 59.3 & 2.5 & 24.2 & 0.52 \\
Dom-kf & 97243 & 10.54 & 11.71 & 6.3 & 39.8 & 1.2 & 11.2 & 0.70 \\
NE-mod & 13624 & 11.21 & 15.67 & 11.4 & 63.9 & 4.5 & 39.8 & 0.57 \\
NE-kf & 13624 & 11.21 & 13.03 & 7.0 & 41.7 & 1.8 & 16.3 & 0.68 \\
SE-mod & 15133 & 11.37 & 13.48 & 7.2 & 44.9 & 2.1 & 18.6 & 0.54 \\
SE-kf & 15133 & 11.37 & 12.29 & 5.2 & 33.4 & 0.9 & 8.1 & 0.66 \\
UM-mod & 16874 & 11.96 & 16.12 & 9.9 & 56.0 & 4.2 & 34.8 & 0.56 \\
UM-kf & 16874 & 11.96 & 13.30 & 6.3 & 37.2 & 1.3 & 11.2 & 0.69 \\
LM-mod & 10936 & 10.17 & 13.09 & 9.4 & 65.2 & 2.9 & 28.8 & 0.39 \\
LM-kf & 10936 & 10.17 & 11.44 & 5.6 & 40.2 & 1.3 & 12.5 & 0.59 \\
RM-mod & 10030 & 8.74 & 11.46 & 9.4 & 70.5 & 2.7 & 31.1 & 0.41 \\
RM-kf & 10030 & 8.74 & 9.79 & 5.9 & 43.8 & 1.1 & 12.1 & 0.68 \\
PC-mod & 17857 & 12.28 & 11.38 & 10.1 & 52.7 & -0.9 & -7.4 & 0.58 \\
PC-kf & 17857 & 12.28 & 12.80 & 7.6 & 38.9 & 0.5 & 4.2 & 0.75 \\
\hline
\end{tabular}

Table 2. Regional summary of discrete statistics for raw model and KF bias-adjusted daily mean $\mathrm{PM}_{2.5}$ forecasts during 2007 warm season.

\begin{tabular}{lcccccrrr}
\hline TYPE & $\mathrm{N}$ & $\begin{array}{c}\text { Obs. mean } \\
\left(\mu \mathrm{g} / \mathrm{m}^{3}\right)\end{array}$ & $\begin{array}{c}\text { Mod. mean } \\
\left(\mu \mathrm{g} / \mathrm{m}^{3}\right)\end{array}$ & $\begin{array}{r}\text { RMSE } \\
\left(\mu \mathrm{g} / \mathrm{m}^{3}\right)\end{array}$ & $\begin{array}{r}\text { NME } \\
(\%)\end{array}$ & $\begin{array}{r}\text { MB } \\
\left(\mu \mathrm{g} / \mathrm{m}^{3}\right)\end{array}$ & $\begin{array}{r}\text { NMB } \\
(\%)\end{array}$ & $r$ \\
\hline Dom-mod & 57319 & 12.51 & 11.83 & 8.4 & 46.0 & -0.7 & -5.4 & 0.52 \\
Dom-kf & 57319 & 12.51 & 12.99 & 6.3 & 34.1 & 0.5 & 3.8 & 0.72 \\
NE-mod & 8097 & 14.97 & 13.16 & 8.7 & 41.1 & -1.8 & -12.1 & 0.61 \\
NE-kf & 8097 & 14.97 & 15.20 & 7.1 & 35.0 & 0.2 & 1.5 & 0.74 \\
SE-mod & 8868 & 17.30 & 13.19 & 9.6 & 36.9 & -4.1 & -23.8 & 0.49 \\
SEkf & 8868 & 17.30 & 17.26 & 7.8 & 29.3 & -0.0 & -0.2 & 0.61 \\
UM-mod & 10120 & 15.17 & 13.40 & 7.3 & 35.3 & -1.8 & -11.7 & 0.62 \\
UM-kf & 10120 & 15.17 & 15.20 & 6.2 & 30.0 & 0.0 & 0.2 & 0.72 \\
LM-mod & 6280 & 12.78 & 11.68 & 9.1 & 52.8 & -1.1 & -8.6 & 0.30 \\
LM-kf & 6280 & 12.78 & 13.53 & 6.3 & 37.1 & 0.8 & 5.9 & 0.51 \\
RM-mod & 5869 & 8.60 & 10.06 & 8.2 & 63.1 & 1.5 & 17.0 & 0.25 \\
RM-kf & 5869 & 8.60 & 9.46 & 5.8 & 40.8 & 0.9 & 10.0 & 0.48 \\
PC-mod & 10062 & 9.34 & 11.60 & 8.4 & 59.9 & 2.3 & 24.2 & 0.52 \\
PC-kf & 10062 & 9.34 & 10.40 & 5.4 & 35.3 & 1.1 & 11.4 & 0.76 \\
\hline
\end{tabular}

\subsection{Systematic/unsystematic errors and performance over concentration bins}

The RMSE can be further decomposed into its systematic and unsystematic components (Willmott, 1981) based on the least-square linear regression relationship between forecast values and observations (Kang et al., 2008). The boxplots in Fig. 10 show the distribution of the RMSE, and its systematic (RMSEs) and unsystematic (RMSEu) components of the predicted daily mean $\mathrm{PM}_{2.5}$ for the raw model and KF forecasts across all the stations within the continental US domain. Shown in the boxplots are the first quartile (lower border of the box), the third quartile (upper border of the box), and the median (the central line) values of the distributions. The whiskers represent the 1.5 IQR (inter-quartile range). The decomposition of the RMSE displays different error characteristics for $\mathrm{PM}_{2.5}$ relative to those noted previously for $\mathrm{O}_{3}$ forecasts (Kang et al., 2008). First, for the raw model forecasts, while systematic errors were larger than the unsystematic components for $\mathrm{O}_{3}$, the converse is noted for $\mathrm{PM}_{2.5}$ forecasts. The larger contribution of unsystematic errors to the $\mathrm{PM}_{2.5}$ RMSE not only reflect the bigger uncertainty in the emissions inventory used and in our understanding of the relevant atmospheric processes, but also the local-level variability in the predominantly urban AIRNOW measurement network. The application of the KF bias adjustment helps 

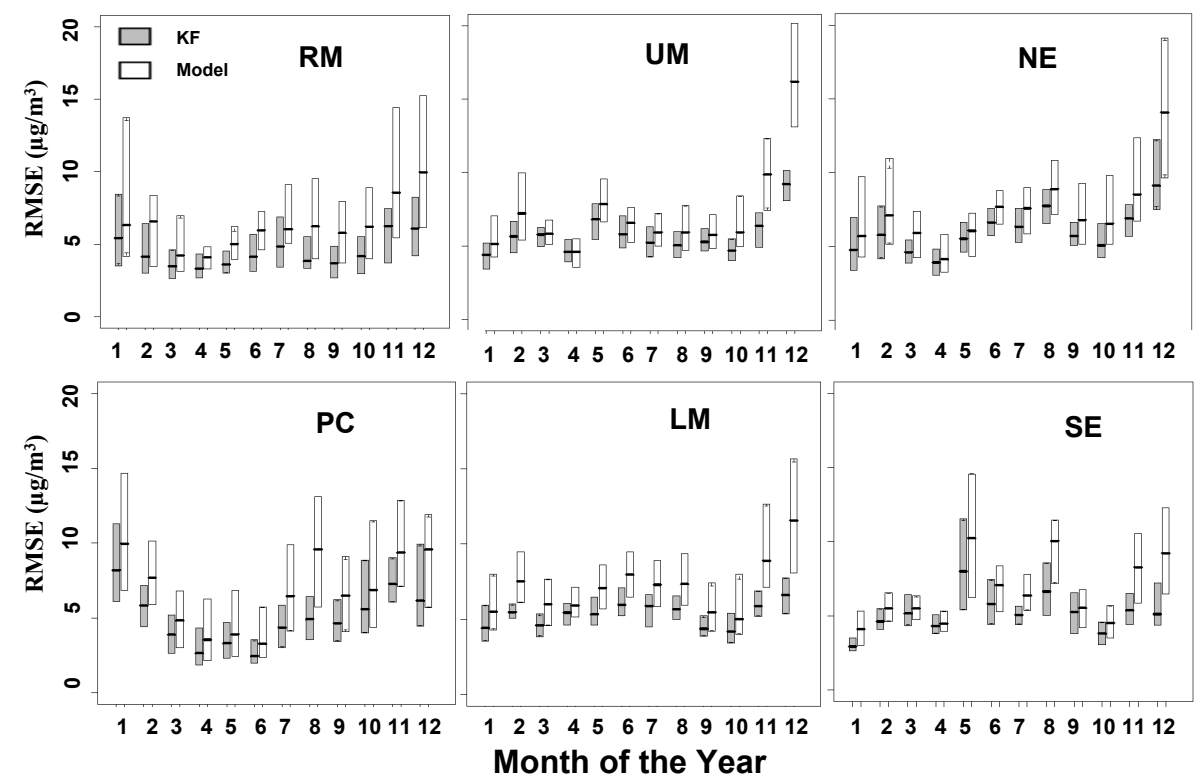

Fig. 7. Monthly box plots (only 25 th and 75 th percentiles and median values are shown) of RMSE values of the daily mean $\mathrm{PM}_{2.5}$ concentrations $\left(\mu \mathrm{g} / \mathrm{m}^{3}\right)$ for the raw model forecasts and KF bias-adjusted forecasts for all the sub-regions.
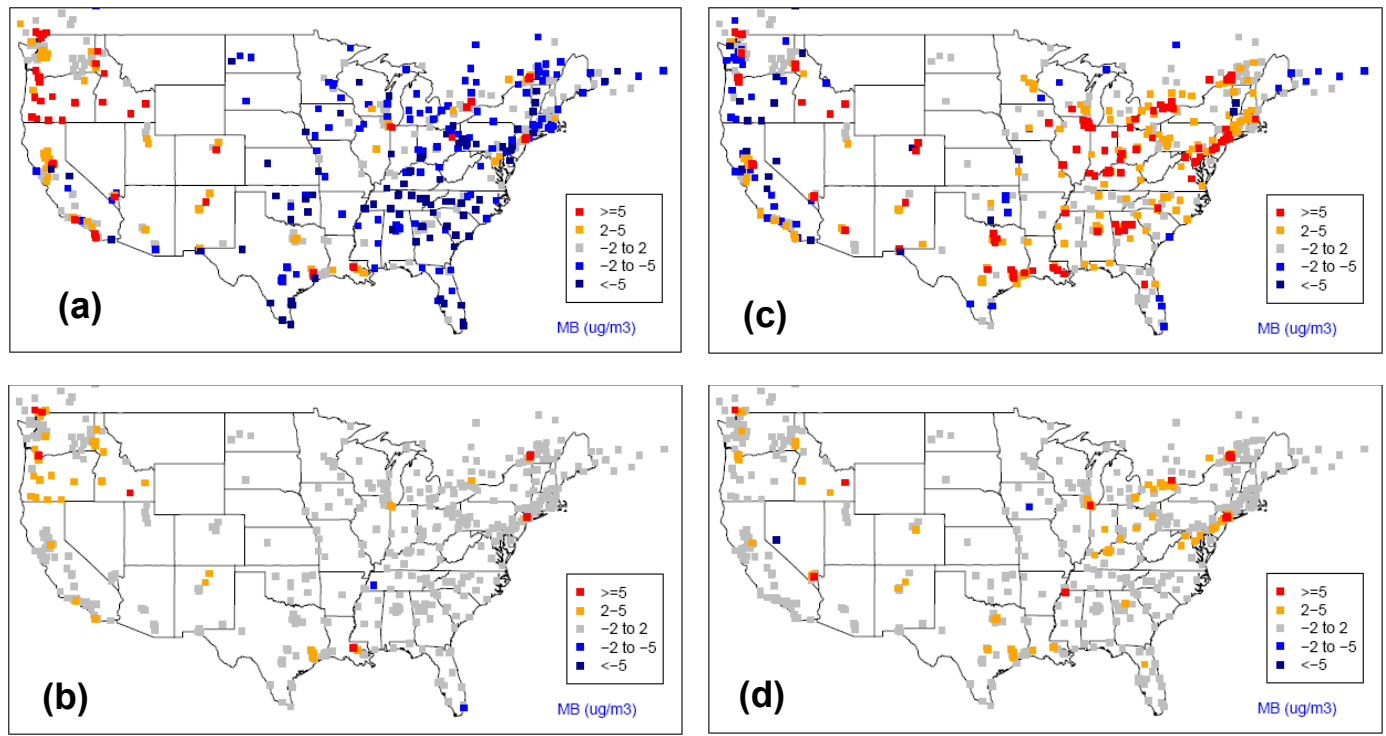

Fig. 8. Mean Bias $\left(\mathrm{MB}, \mu \mathrm{g} / \mathrm{m}^{3}\right)$ at each location within the continental US Domain: (a) raw model during warm season, (b) KF biasadjustment during warm season, (c) raw model during cool season, and (d) KF bias-adjustment during cool season.

reduce both the unsystematic and systematic errors in $\mathrm{PM}_{2.5}$ forecasts.

To further examine the performance of the KF biasadjustment technique over different concentration ranges, Fig. 11 displays the forecast RMSE and MB values as a function of observed concentrations for both the warm and cool seasons. During the warm season (Fig. 11a), when observed $\mathrm{PM}_{2.5}$ concentrations were less than $10 \mu \mathrm{g} / \mathrm{m}^{3}$, the KF bias-adjustment technique was unable to reduce RMSE val- ues compared to the raw model forecasts, though the distributions were narrower. This may in part be attributed to the fact that during the warm season, the weather conditions tend to be more variable (more convective weather conditions) than those during the cool season and lower concentrations are often associated with precipitation processes, and the raw model generally has difficulty to accurately simulate these weather conditions, resulting in larger unsystematic errors in the prediction of $\mathrm{PM}_{2.5}$ concentrations. When the 

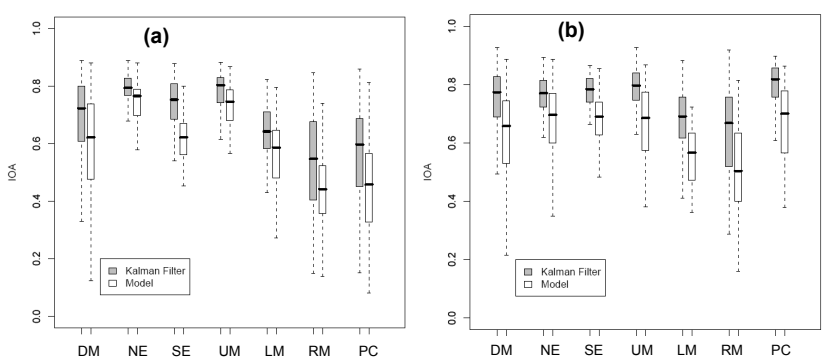

Fig. 9. Box plots of index of agreement (IOA) of daily mean $\mathrm{PM}_{2.5}$ $\left(\mu \mathrm{g} / \mathrm{m}^{3}\right)$ for the raw model (MOD) forecasts and KF bias-adjusted forecasts over the domain (DM) and across all sub-regions during (a) warm season and (b) cool season.

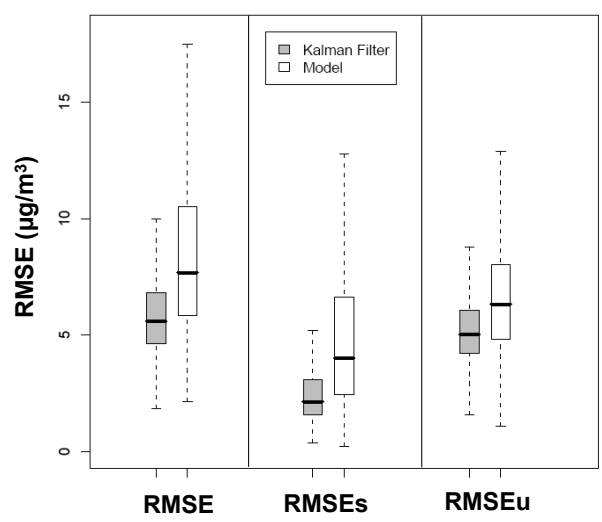

Fig. 10. Box plots of RMSE and decomposed RMSE (systematic, RMSEs; unsystematic, RMSEu) values of the daily mean $\mathrm{PM}_{2.5}$ concentrations $\left(\mu \mathrm{g} / \mathrm{m}^{3}\right)$ for the raw model forecasts and KF biasadjusted forecasts.

observed $\mathrm{PM}_{2.5}$ concentrations were larger than $10 \mu \mathrm{g} / \mathrm{m}^{3}$, the RMSE values associated with KF forecasts were much smaller in both the mean values and the distributions compared to the raw model forecasts. In contrast, during the cool season (Fig. 11b), the KF forecasts performed better than the raw model forecasts across all the concentration bins. Examination of the MB distributions over the observed concentration bins (Fig. 11c and d) reveals that the raw model over-predicted at lower concentrations and under-predicted at higher concentrations, which is similar to the raw model performance for $\mathrm{O}_{3}$ forecasts (Kang et al., 2008). The underprediction at higher concentration bins for $\mathrm{PM}_{2.5}$ forecasts during the warm season was more severe than that during the cool season. In general, the KF forecasts were able to adjust the MB towards zero over all the concentration bins for both seasons.

\subsection{Categorical performance}

It is equally important to evaluate the performance of an air quality forecast system using the categorical metrics, because for the general public, it is more important to know if

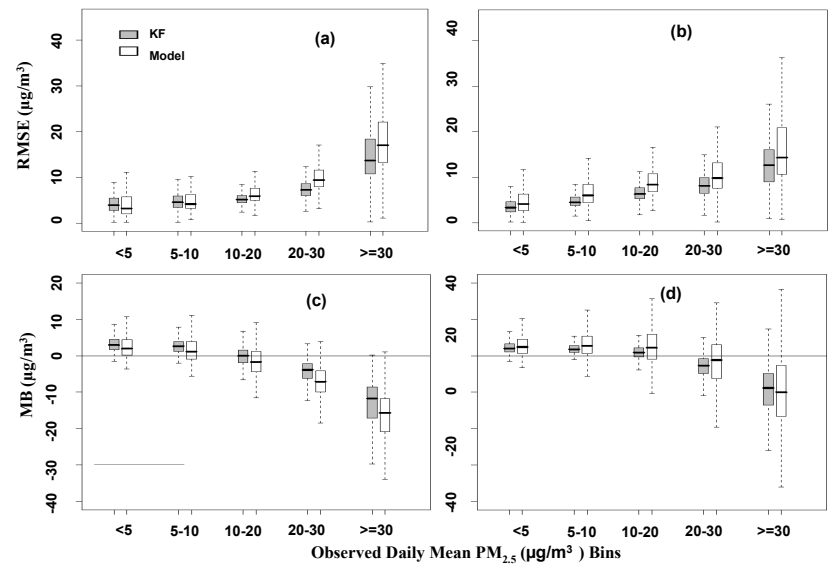

Fig. 11. (a and b) RMSE and (c and d) mean bias (MB) values over observed daily mean $\mathrm{PM}_{2.5}$ concentration $\left(\mu \mathrm{g} / \mathrm{m}^{3}\right)$ bins for the raw model forecasts and the KF bias-adjusted forecasts. The sample sizes for each bin from small to large are 28203, 17526, 19855, 6855, 2716 (Warm Season) and 34072, 36402, 30697, 6771, 2502 (Cool Season).

the NAQFC system could simulate the occurrences of an exceedance or non-exceedance. Categorical evaluations for $\mathrm{O}_{3}$ forecasts have been extensively performed in the past (Kang et al., 2005; Eder et al., 2006, 2009), but similar assessments for $\mathrm{PM}_{2.5}$ forecasts have been limited. Figure 12 displays the false alarm ratio (FAR; also known as probability of false alarm) and hit rate $(\mathrm{H}$; also known as probability of detection) (see Kang et al., 2005; Barnes et al., 2009) for the raw model and KF bias-adjusted daily mean $\mathrm{PM}_{2.5}$ forecasts for each of the sub-regions during both the warm and cool seasons. An exceedance threshold value of $35 \mu \mathrm{g} / \mathrm{m}^{3}$ for the 24 $\mathrm{h}$ mean $\mathrm{PM}_{2.5}$, based on the US National Ambient Air Quality Standard (NAAQS) for $\mathrm{PM}_{2.5}$ is used. As seen in Fig. 12, the FAR values associated with the raw model forecasts were similar $(\sim 85 \%)$ for both seasons over the entire domain, but the $\mathrm{H}$ values varied from less than $10 \%$ during the warm season to greater than $30 \%$ during the cool season. For the KF forecasts, the FAR values were reduced by more than $20 \%$ during both seasons, and the $\mathrm{H}$ values have more than doubled during the warm season and were increased by about $20 \%$ during the cool season for the entire domain. Compared to the raw model forecasts, the KF forecasts reduced the FAR values across all the sub-regions, with differing magnitudes and increased the $\mathrm{H}$ values for all the sub-regions except for the LM and RM in the warm season and he UM in the cool season. In general, the $\mathrm{H}$ values were higher during the cool season than those during the warm season for both the raw model forecasts and the KF forecast, while the FAR values didn't differ significantly. 
(a) Warm Season

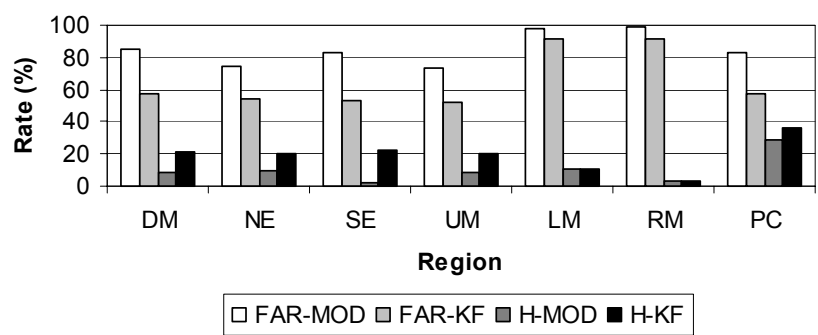

(b) Cool Season

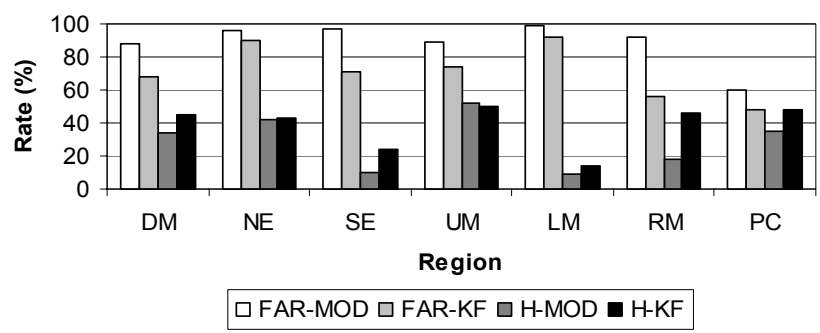

Fig. 12. False alarm ratio (FAR) and hit rate $(H)$ for the daily mean $\mathrm{PM}_{2.5}$ forecasts by the raw model and the KF bias-adjustment over the domain (DM) and all the sub-regions during (a) warm season and (b) cool season: FAR-MD, FAR associated with raw model forecasts; FAR-KF, FAR associated with KF forecasts; H-MD, H associated with raw model forecasts; and $\mathrm{H}-\mathrm{KF}, \mathrm{H}$ associated with KF forecasts.

\section{Summary}

The Kalman filter bias-adjustment technique has been applied to post-process raw $\mathrm{PM}_{2.5}$ air quality forecasts over the continental US domain during the year of 2007 at hourly $\mathrm{PM}_{2.5}$ monitoring sites. Though the application and analysis were conducted on archived $\mathrm{PM}_{2.5}$ model forecast output, the methodology is easily adopted for real-time applications. To facilitate performance evaluation, the continental US portion of the domain was divided into six sub-regions and the year was split into a cool season and a warm season to examine spatial and seasonal characteristics of the performance of the method. The evaluation of raw model performance suggests that the daily mean $\mathrm{PM}_{2.5}$ concentrations were generally over-predicted over the eastern part of the domain during the cool season and under-predicted during the warm season; in contrast, the opposite is true for the western part of the domain, i.e., the daily mean $\mathrm{PM}_{2.5}$ concentrations were typically under-predicted along the Pacific Coast during the cool season and over-predicted during the warm season; the Rocky Mountain region is an exception where the daily mean $\mathrm{PM}_{2.5}$ concentrations were over-predicted through the year.

The KF bias-adjustment technique significantly improved the $\mathrm{PM}_{2.5}$ forecasts for locations with hourly $\mathrm{PM}_{2.5}$ monitors as revealed by reductions in errors and biases, and higher correlation coefficients throughout the year and across the entire model domain. The analysis also shows that the KF bias adjustment can quickly respond to transitions from one regime to another during the transition of seasons or model changes.

Analysis of RMSE and MB as a function of observed concentrations suggests that the KF method significantly reduces the raw model error and bias across all concentration ranges except at lower concentration bins during the warm season. However, the significant reductions in error and bias at the moderate-high concentration ranges helps improve the ability to predict exceedances, a feature desirable for air quality forecasting. The effectiveness and benefits of biasadjustment of $\mathrm{PM}_{2.5}$ model forecasts is also reflected in the categorical evaluations; the KF bias-adjustment technique improved the categorical evaluation metrics significantly by reducing the false alarm ratio and increasing the hit rate for almost all the regions during both the cool and warm seasons.

It should be pointed out that the performance of biasadjusted forecasts is dependent on the performance of the raw model to which the bias-adjustment technique is applied. Because of the complexity in $\mathrm{PM}_{2.5}$ composition, formation, and distribution, it is even more critical for the raw model to provide a stable and well-behaved basis to make biasadjusted forecasts more reliable. This bias-adjusted forecast study was based on the total mass of $\mathrm{PM}_{2.5}$. If the components of $\mathrm{PM}_{2.5}$ could be bias-adjusted separately, the results may be further improved than those derived from the bias-adjustment of the total $\mathrm{PM}_{2.5}$ mass performed in this study. However, the lack of real-time measurements of speciated $\mathrm{PM}_{2.5}$ hampers the use of $\mathrm{KF}$ adjustments on individual species. Improvements in the representation of fine particulate matter emissions as well as physical and chemical processes regulating sources and sinks in atmospheric models are expected as a result of on-going research over the next several years. Nevertheless, our analysis indicates that despite the current uncertainties in the representation of atmospheric processes dictating the distribution of ambient $\mathrm{PM}_{2.5}$, the KF bias-adjustment techniques can be used to improve the reliability of short term $\mathrm{PM}_{2.5}$ forecasts from such models and, consequently, help in issuance of air-qualitydegradation-related health advisories.

In this study, the KF bias-adjustment technique is only applied at discrete points, i.e., at location of the monitors. Further research is needed to extend this technique for the development of bias-corrected spatial maps (i.e., also at location where no monitor information is available) for surfacelevel $\mathrm{PM}_{2.5}$ distributions. Since surface-level $\mathrm{PM}_{2.5}$ concentrations are influenced by local forcing associated with several meteorological drivers and spatially-heterogeneous emissions, information on the spatial representativeness of the individual measurements and, consequently, the adjusted bias is critical to the extension of this method presented here to develop bias-adjusted spatial maps of $\mathrm{PM}_{2.5}$ forecast. 
Acknowledgements. The authors are grateful to Brian Eder for his constructive and insightful comments on initial drafts of this manuscript. We thank Drs. Luca Delle Monache and Roland B. Stull for providing their original Kalman filter codes. The United States Environmental Protection Agency through its Office of Research and Development funded and managed the research described here. It has been subjected to Agency's administrative review and approved for publication.

Edited by: D. Lunt

\section{References}

Appel, K. W., Bhave, P. V., Gilliland, A. B., Sarwar, G., and Roselle, S. J.: Evaluation of the community multiscale air quality (CMAQ) model version 4.5: Sensitivities impacting model performance; Part II-particulate matter, Atmos. Environ., 42, 60576066, doi:10.1016/j.atmosenv.2008.03.036, 2008.

Barnes, L. R., Schultz, D. M., Gruntfest, E. C., Hayden, M. H., and Benight, C. C.: Corrigendum: False Alarm Rate or False Alarm Ratio, Weather Forecast., 24, 1452-1454, doi:10.1175/2009WAF2222300.1, 2009.

Black, T.: The new NMC mesoscale Eta Model: Description and forecast examples, Weather Forecast., 9, 265-278, 1994.

Byun, D. W. and Schere, K. L.: Review of the governing equations, computational algorithms, and other components of the Models3 Community Multiscale Air Quality (CMAQ) modeling system, Appl. Mech. Rev., 59, 51-77, 2006.

Delle Monache, L., Nipen, T., Deng, X., Zhou, Y., and Stull, R.: Ozone ensemble forecasts: 2. A Kalman filter predictor bias correction, J. Geophys. Res., 111, D05308, doi:10.1029/2005JD006311, 2006.

Delle Monache, L., Wilczak, J., Mckeen, S., Grell, G., Pagowski, M., Peckham, S., Stull, R., McHenry, J., and McQueen, J.: A Kalman-filter bias correction method applied to deterministic, ensemble averaged, and probabilistic forecasts of surface ozone, Tellus Ser. B, 60, 238-249, doi:10.111/j.16000889.2007.00332.x, 2008.

Donahue, N. M., Robinson, A. L., and Pandis, S. N.: Atmospheric particulate matter: from smoke to secondary organic aerosol, Atmos. Environ., 43, 94-106, doi:10.1016/j.atmosenv.2008.09.055, 2009.

Eder, B., Davis, J., and Bloomfield, P.: A characterization of the spatiotemporal variation of non-urban ozone in the eastern United States, Atmos. Environ., 27A, 2645-2668, 1993.

Eder, B., Kang, D., Mathur, R., Yu, S., and Schere, K.: An operational evaluation of the Eta-CMAQ air quality forecast model, Atmos. Environ., 40, 4894-4905, 2006.

Eder, B., Kang, D., Mathur, R., Pleim, J., Yu, S., Otte, T., and Pouliot, G.: A performance evaluation of the national air quality forecast capability for the summer of 2007, Atmos. Environ., 43, 2312-2320, doi:10.1016/j.atmosenv.2009.01.033, 2009.

Eatough, D. J., Long, R. W., Modey, W. K., and Eatough, N. L.: Semi-volatile secondary organic aerosol in urban atmospheres: meeting a measurement challenge, Atmos. Environ., 37, 12771292, doi:10.1016/S1352-2130(02)01020-8, 2003.
Gego, E. L., Porter, P. S., Irwin, J. S., Hogrefe, C., and Rao, S. T.: Assessing the comparability of ammonium, nitrate and sulfate concentrations measured by three air quality monitoring networks, Pure Appl. Geophys., 162, 1919-1939, doi:10.1007/s00024-005-2698-3, 2005.

Glahn, H. R. and Lowry, D. A.: The use of model output statistics (MOS) in objective weather forecasting, J. Appl. Meteor., 11, 1203-1211, 1972.

Grover, B. D., Kleinman, M., Eatough, N. L., Eatough, D. J., Hopke, P. K., Long, R. W., Wilson, W. E., Meyer, M. B., and Ambs, J. L.: Measurement of total $\mathrm{PM}_{2.5}$ mass (nonvolatile plus semivolatile) with the Filter Dynamic Measurement System tapered element oscillating microbalance monitor, J. Geophys. Res., 110, D07S03, doi:10.1029/2004JD004995, 2005.

Kalman, R. E.: A new approach to linear filtering and prediction problems, J. Basic Eng., 82, 35-45, 1960.

Kang, D., Mathur, R., Rao, S. T., and Yu, S.: Bias adjustment techniques for improving ozone air quality forecasts, J. Geophys. Res., 113, D23308, doi:10.1029/2008JD010151, 2008.

Kang, D., Eder, B. K., Stein, A. F., Grell, G. A., Peckham, S. E., and McHenry, J.: The New England air quality forecasting pilot program: Development of an evaluation protocol and performance benchmark, J. Air Waste Manage. Assoc., 55, 1782-1796, 2005.

Mathur, R., Yu, S., Kang, D., and Schere, K. L.: Assessment of the wintertime performance of developmental particulate matter forecasts with the Eta-Community Multiscale Air Quality modeling system, J. Geophys. Res., 113, D02303, doi:10.1029/2007JD008580, 2008.

McHenry, J. N., Ryan, W. F., Seaman, N. L., Coats Jr., C. J., Pudykiewicz, J., Arunachalam, S., and Vukovich, J. M.: A realtime Eulerian photochemical model forecast system: Overview and initial ozone forecast performance in the northeast U. S. corridor, B. Am. Meteorol. Soc., 85, 525-548, 2004.

McKeen, S., Wilczak, J., Grell, G., et al.: Assessment of an ensemble of seven real-time ozone forecasts over eastern North America during the summer of 2004, J. Geophys. Res., 110, D21307, doi:10.1029/2005JD005858, 2005.

McKeen, S., Chung, S. H., Wilczak, J., et al.: Evaluation of several PM2.5 forecast models using data collected during the ICARTT/NEAQS 2004 field study, J. Geophys. Res., 112, D10S20, doi:10.1029/2006JD007608, 2007.

McMurry, P. A.: A review of atmospheric aerosol measurements, Atmos. Environ., 34, 1959-1999, 2000.

National Research Council: Research Priorities for Particulate Matter 1: Immediate Priorities and a Long-Range Research Portfolio, Natl. Acad. Press, Washington, D.C., 1998.

Otte, T. L., Pouliot, G., Pleim, J. E., et al.: Linking the Eta Model with the Community Multiscale Air Quality (CMAQ) modeling system to build a national air quality forecasting system, Weather Forecast., 20, 367-384, 2005.

Rogers, E., Black, T. L., Deaven, D. G., DiMego, G. J., Zhao, O., Baldwin, M., Junker, N. W., and Lin, Y.: Changes to the operational "early" Eta Analysis/Forecast System at the National Centers for Environmental Prediction, Weather Forecast., 11, 391413, 1996. 
Schwede, D., Pouliot, G., and Pierce, T.: Changes to the Biogenic Emissions Inventory System Version 3 (BEIS3) at the 4th Annual CMAS Models-3 User's Conference, 26-28 September 2005, Chapel Hill, NC, USA, 2005.

Wilczak, J., McKeen, S., Djalalova, I., Grell, G., Peckham, S., Gong, W., Bouchet, V., Moffet, R., McHenry, J., Lee, P., Tang, Y., and Carmichael, G. R.: Bias-corrected ensemble and probabilistic forecasts of surface ozone over eastern North America during the summer of 2004, J. Geophys. Res., 111, D23S28, doi:10.1029/2006JD007598, 2006.
Willmott, C. J.: On the validation of models, Phys. Geogr., 2, 184194, 1981.

Yu, S., Mathur, R., Schere, K., Kang, D., Pleim, J., Young, J., Tong, D., Pouliot, G., Mckeen, S., and Rao, S. T.: Evaluation of realtime PM2.5 forecasts and process analysis for PM2.5 formation over the eastern United States using Eta-CMAQ forecast model during the 2004 ICARTT study, J. Geophys. Res., 113, D06204, doi:10.1029/2007JD009226, 2008. 